\title{
Cell Surface Heart Shock Proteins and their Role in Cancer
}

\section{Gopal $\mathrm{A}^{1}$ and Gopal $\mathrm{U}^{2 *}$}

${ }^{1}$ Department of Biotechnology, Center for Biotechnology, Anna University, India

2Department of Pathology, Duke University Medical Center, USA

*Corresponding author: Udhayakumar Gopal, Department of Pathology, Duke

University Medical Center, Durham, NC, USA, Tel: 91-684-8986, Fax 91-684-8689; E-mail: udhayakumar.gopal@duke.edu

\section{Review Article}

Volume 1 Issue 1

Received Date: April 03, 2017

Published Date: May 30, 2017

DOI: $10.23880 /$ cprj-16000104

\section{Abstract}

Heat-shock proteins are commonly considered to be an intracellular molecular chaperone which performs multitude of functions like cytoprotective and cellular housekeeping functions. However many of these chaperones translocate into the surface of the cells particularly during stress induced conditions like hypoxia, UV-radiation, chemotherapy, drugs and microbial stimuli. Once on the cell surface or in the extracellular space, the heat shock proteins functions like receptors for wide variety of ligands by which it regulates signaling, proliferation, invasion, apoptosis, inflammation and immunity. Thus, cell-surface Heat-shock proteins may play a unique role in tumor metastasis, distinct from but perhaps overlapping with its intracellular function. The discovery of cell surface Heat shock proteins in cancer cells and cells undergoing stress presents a novel therapeutic strategy.

Keywords: Heat shock Protein; Cell surface; Cancer; ER Stress; CS-HSP90; CS-GRP78

\section{Introduction}

Molecular Chaperones are proteins that permit the maturation and correct folding of most of the proteome $[1,2]$. As such, they are found in all cellular organisms and seem essential for cellular life. Protein folding seems to require chaperones from a number of different gene families that appear to function at various stages in a concerted folding cascade. These proteins belong to the small heat-shock protein (HSP) family including HSP27 and the large 70Kda HSP family including HSP70 as well as HSP60, HSP90, and HSP110 families [3]. The acronym HSP is derived from the early findings that some of these proteins are massively induced during proteotoxic stresses such as heat shock [4]. Thus the canonical functions of the HSP chaperones are in the folding of proteins during mRNA translation and in responding to protein unfolding crises in stressed cells [5].

\section{Cell-Surface HSPs and Cancer Metastasis}

However, HSPs also appear to possess functions outside the realm of protein folding, some of them acquired when they are released from cells to become Cell Surface (CS)-HSPs [5-8]. HSPs have been observed in serum from human patients, pointing to their existence outside of cells in living organisms [9]. The properties of extracellular HSPs have now expanded from immune response to major intercellular signaling molecules in biology and medicine. In this review we describe only the functions of CS-HSPs in cancer and their possible role in various pathologies.

As summarized in (Table 1) it has been shown that HSP are expressed on the cell surface and that these proteins are crucial for cancer progression. HSP25 a murine homolog of human HSP27, has been shown to regulate metastasis [10]. Surface expression of HSP25 correlates with enhanced breast cancer growth in vivo and cells expressing higher levels of HSP25 on the cell surface 
display enhanced metastatic potential in vitro and enhanced metastatic activity in vivo. HSP70, a major stress- inducible chaperone is expressed at the cell surface of melanoma metastases but not in normal skin fibroblasts [11]. Cell surface expression of HSP70 is observed to be significantly higher in clinically advanced oral tumors [12], suggesting that cell surface HSP70 might be crucial for tumor progression. In contrast, CS-HSP70 seems to inhibit metastasis in breast cancer cells [10]. This discrepancy may be due to the difference in cell
type.HSP90 identified on the cell-surface is crucial for cell motility in neuronal, glioma and melanoma cells [7-34]. CS-HSP90 interacts with low-density lipoprotein receptor-related protein (LRP1/CD91) to induce a number of pro-motility signaling cascades that are essential for wound healing [15]. In glioma cells extracellular HSP90 promotes interaction of LRP1 with ephrin type-A receptor tyrosine kinase 2 (EPHA2) by activating AKT signaling which induces pro-motility function [7].

\begin{tabular}{|c|c|c|c|}
\hline ER HSPs & Over expression in diseases & Potential role & Reference \\
\hline HSP47 & Oral Cancer & Migration and Invasion & {$[25]$} \\
\hline GRP78 & Prostate, Glioblastoma & Tumor proliferation, gene activation & {$[26,27]$} \\
\hline ERP57 & Breast and Gastric cancer & Tumor proliferation & {$[28,29]$} \\
\hline PDI & Prostate and Lung Cancer & Migration & {$[30,31]$} \\
\hline GP96 & Ovarian and Pancreatic cancer & Immune response & {$[32]$} \\
\hline Calreticulin & Melanoma & Migration & {$[34]$} \\
\hline
\end{tabular}

Table 1: Summary of CS-HSPs in Cancer.

\section{Endoplasmic Reticulum (ER) Chaperones}

The Endoplasmic reticulum (ER) is one of many specialized organelles in the cell with diverse and apparently ever expanding functionality. Two of the major functions of the ER, namely calcium sequestration [16] and the correct assembly, folding and secretion of glycoproteins were established over the decades [17-19]. In ER number of proteins functions like heat shock proteins which are HSP47, GRP78, ERP57, protein disulfide isomerase (PDI), gp96 and calreticulin [20]. During chemical or physical cell stress chaperones relocate to the cell surface where it's associated with various diseases particularly cancer (Table 2). Over expression of chaperones on the cell surface to cope with increased ER stress due to malignancy. CS-ER chaperones might be simply a biomarker and we highlight their direct role in the spread of tumors by promoting cell proliferation [21], migration [22] and metastasis [23,24].

\begin{tabular}{|c|c|c|c|}
\hline HSPs & Over expression in diseases & Potential role & Reference \\
\hline HSP25 & Breast Cancer & Increases metastasis & {$[10]$} \\
\hline HSP70 & Oral Tumor, Melanoma & Tumor proliferation, Metastasis & {$[11,12]$} \\
\hline HSP90 & Glioblastoma, Fibroblast & Migration, Wound healing & {$[7,15]$} \\
\hline
\end{tabular}

Table 2: Summary of CS-ER HSPs in Cancer.

\section{Cell Surface ER Chaperones and Cancer}

HSP47, which is known as a rheumatoid arthritisrelated antigen, has been shown to be expressed at the cell surface, and oral cancer cells expressing high levels of surface HSP47 display low invasive activity, suggesting that HSP47 has an inhibitory effect on cell migration/invasion [25]. Thus, may distinct chaperone proteins might be expressed at the cell surface, and have diverse effects on cell motility, invasion, and cancer progression.GRP78 expresses on the cell surface where it functions as a multifunctional receptor for a wide variety of ligands to mediate proliferative signaling in various human cancers. Though the GRP78 forms complexes with other proteins on the cell surface, GRP78 is reported to mediate tumor cell signal transduction. Cell Surface GRP78 is also an angiogenic receptor on endothelial cells by increasing its proliferation, migration and tube formation [26]. Moreover, CS-GRP78 is required for the transcriptional activation of a subset of c-Myc target genes and cell transformation [27]. It suggests that CSGRP78 function not only as a signaling mediator but also activates transcription factors in tumor growth.

ERP57 increases in the cell surface regulate EGF receptor signaling and internalization in breast cancer cells. Moreover secretion of ERP57 is essential for matrix accumulation and prognostic significance in gastric 
cancer $[28,29]$. CS-PDI is upregulated in CNS cancers, lymphoma's ovarian, lung and prostate cancer. Furthermore it regulates multiple important biological processes like injury response and it promotes glioma cell migration [30,31]. GP96, the HSP90 homology in endoplasmic reticulum, has been shown to be expressed at the cell surface only in tumor cells [32], and it was the first chaperone described to play a role in the induction of anti-tumor immune responses. Several phase I and phase II clinical trials are currently ongoing using a GP96peptide complex as a cancer vaccine. The role of GP96 in mediating immune responses was well summarized in a recent review [33]. Calreticulin, an endoplasmic reticulum chaperone is also expressed at the cell surface. Calreticulin has been identified as the cell surface lectin responsible for triggering cell spreading of melanoma cells [34]. Treatment of cells with calreticulin antibody inhibited laminin-dependent cell spreading, suggesting that calreticulin is crucial for cell motility and that it might participate in integrin signaling. Indeed, it is reported that calreticulin is associated with integrin $\alpha$ $2 / \beta-1$ on the platelet surface and that it modulates ligand interaction with integrin's [35].

\section{Conclusion}

Chaperone molecules play a number of specific roles related to protein processing within the cell. It's also well known that HSPs play a central role in cancer development and progression. However, new knowledge indicates that a select number of chaperones in the extracellular environment can play a role in tumor growth as well as invasion and metastasis. Therefore it is crucial to understand how various post-translational modified forms of chaperones are release from cells under resting and stressed conditions and how the released chaperones exerts their proliferative and cell survival responses. The precise nature of the functions and molecular mechanisms of CS-HSPs is crucial for enhancing the accuracy of cancer diagnosis as well as for developing more effective therapeutic agents.

\section{References}

1. Ellis RJ (2007) Protein misassembly: macromolecular crowding and molecular chaperones. Adv Exp Med Biol 594: 1-13.

2. Haldar S, Gupta AJ, Yan X, Milicic G, Hartl FU, et al. (2015) Chaperonin-Assisted Protein Folding: Relative Population of Asymmetric and Symmetric GroEL: GroES Complexes. J Mol Biol 427(12): 2244-2255.
3. Lindquist S, Craig EA (1988) The heat-shock proteins. Ann Rev Genet 22: 631-677.

4. Richter K, Haslbeck M, Buchner J (2010) The heat shock response: life on the verge of death. Molecular cell 40(2): 253-266.

5. Salari S, Seibert T, Chen YX, Hu T, Shi C, et al. (2013) Extracellular HSP27 acts as a signaling molecule to activate NF-kappaB in macrophages. Cell stress chaperones 18(1): 53-63.

6. Tian J, Guo X, Liu XM, Liu L, Weng QF, et al. (2013) Extracellular HSP60 induces inflammation through activating and up-regulating TLRs in cardiomyocytes. Cardiovasc Res 98(3): 391-401.

7. Gopal U, Bohonowych JE, Lema Tome C, Liu A, Garrett Mayer E, et al. (2011) A novel extracellular Hsp90 mediated co-receptor function for LRP1 regulates EphA2 dependent glioblastoma cell invasion. PloS One 6(3): e17649.

8. Mambula SS, Calderwood SK (2006) Heat shock protein 70 is secreted from tumor cells by a nonclassical pathway involving lysosomal endosomes. J Immunol 1: 177(11): 7849-7857.

9. Wright BH, Corton JM, El Nahas AM, Wood RF, Pockley AG (2000) Elevated levels of circulating heat shock protein 70 (Hsp70) in peripheral and renal vascular disease. Heart and vessels 15(1): 18-22.

10. Bausero MA, Page DT, Osinaga E, Asea A (2004) Surface expression of Hsp25 and Hsp72 differentially regulates tumor growth and metastasis. Tumour Biol 25(5-6): 243-251.

11. Farkas B, Hantschel M, Magyarlaki M, Becker B, Scherer K, et al. (2003) Heat shock protein 70 membrane expression and melanoma-associated marker phenotype in primary and metastatic melanoma. Melanoma Res 13(2): 147-152.

12. Kaur J, Das SN, Srivastava A, Ralhan R (1998) Cell surface expression of $70 \mathrm{kDa}$ heat shock protein in human oral dysplasia and squamous cell carcinoma: correlation with clinicopathological features. Oral Oncol 34(2): 93-98.

13. Sidera K, Samiotaki M, Yfanti E, Panayotou G, Patsavoudi E (2004) Involvement of cell surface HSP90 in cell migration reveals a novel role in the developing nervous system. J Biol Chem 279(44): 45379-45388. 
14. Stellas D, Karameris A, Patsavoudi E (2007) Monoclonal antibody 4C5 immunostains human melanomas and inhibits melanoma cell invasion and metastasis. Clin cancer Res 13(6): 1831-1838.

15. Cheng CF, Fan J, Fedesco M, Guan S, Li Y, et al. (2008) Transforming growth factor alpha (TGFalpha)stimulated secretion of HSP90alpha: using the receptor LRP-1/CD91 to promote human skin cell migration against a TGFbeta-rich environment during wound healing. Mol Cell Biol 28(10): 3344-3358.

16. Mazzarello P, Calligaro A, Vannini V, Muscatello U (2003) The sarcoplasmic reticulum: its discovery and rediscovery. Nat Rev Mol Cell Biol 4(1): 69-74.

17. Krieg UC, Johnson AE, Walter P (1989) Protein translocation across the endoplasmic reticulum membrane: identification by photocross-linking of a 39-kD integral membrane glycoprotein as part of a putative translocation tunnel. J Cell Bio 109(5): 20332043.

18. Deshaies RJ, Schekman R (1987) A yeast mutant defective at an early stage in import of secretory protein precursors into the endoplasmic reticulum. J Cell Biol 105(2): 633-645.

19. Caro LG, Palade GE (1964) Protein Synthesis, Storage, and Discharge in the Pancreatic Exocrine Cell. An Autoradiographic Study. J Cell Biol 20: 473-495.

20. Schwanhausser B, Busse D, Li N, Dittmar G, Schuchhardt J, et al. (2011) Global quantification of mammalian gene expression control. Nature 473(7347): 337-342.

21. Platet N, Cunat S, Chalbos D, Rochefort H, Garcia M (2000) Unliganded and liganded estrogen receptors protect against cancer invasion via different mechanisms. Mol Endocrinol 14(7): 999-1009.

22. Arnaudeau S, Frieden $M$, Nakamura K, Castelbou C, Michalak M, et al (2002) Calreticulin differentially modulates calcium uptake and release in the endoplasmic reticulum and mitochondria. J Biol Chem 277(48): 46696-46705.

23. Chen CN, Chang CC, Su TE, Hsu WM, Jeng YM, et al. (2009) Identification of calreticulin as a prognosis marker and angiogenic regulator in human gastric cancer. Ann Surg Oncol 16(2): 524-533.

24. Lu YC, Chen CN, Wang B, Hsu WM, Chen ST, et al. (2011) Changes in tumor growth and metastatic capacities of J82 human bladder cancer cells suppressed by down-regulation of calreticulin expression. Am J Pathol 179(3): 1425-1433.

25. Hebert C, Norris K, Della Coletta R, Reynolds M, Ordonez J, Sauk JJ. Cell surface colligin/Hsp47 associates with tetraspanin protein CD9 in epidermoid carcinoma cell lines. J Cell Biochem 73(2): 248-258.

26. Gonzalez Gronow M, Selim MA, Papalas J, Pizzo SV (2009) GRP78: a multifunctional receptor on the cell surface. Antioxidants Redox signal 11(9): 2299-2306.

27. Gopal U, Gonzalez Gronow M, Pizzo SV (2016) Activated alpha2-Macroglobulin Regulates Transcriptional Activation of c-MYC Target Genes through Cell Surface GRP78 Protein 291(20): 1090415

28. Gaucci E, Altieri F, Turano C, Chichiarelli S (2013) The protein ERp57 contributes to EGF receptor signaling and internalization in MDA-MB-468 breast cancer cells. J Cell Biochem 114(11): 2461-2470.

29. Leys CM, Nomura S, LaFleur BJ, Ferrone S, Kaminishi $M$, et al. (2007) Expression and prognostic significance of prothymosin-alpha and ERp57 in human gastric cancer. Surgery 141(1): 41-50.

30. Xu S, Sankar S, Neamati N (2014) Protein disulfide isomerase: a promising target for cancer therapy. Drug Discov Today 19(3): 222-240.

31. Xu S, Butkevich AN, Yamada R, Zhou Y, Debnath B, et al. (2012) Discovery of an orally active smallmolecule irreversible inhibitor of protein disulfide isomerase for ovarian cancer treatment. Proc Natl Acad Sci U S A 109(40): 16348-16353.

32. Altmeyer A, Maki RG, Feldweg AM, Heike M, Protopopov VP, et al. (1996) Tumor-specific cell surface expression of the-KDEL containing, endoplasmic reticular heat shock protein gp96. Int J Cancer 69(4): 340-349.

33. Li Z, Dai J, Zheng H, Liu B, Caudill M (2002) An integrated view of the roles and mechanisms of heat shock protein gp96-peptide complex in eliciting immune response. Front Biosci 7: d731-51.

34. White TK, Zhu Q, Tanzer ML (1995) Cell surface calreticulin is a putative mannoside lectin which triggers mouse melanoma cell spreading. The Journal of biological chemistry 270(27): 15926-15929. 
35. Elton CM, Smethurst PA, Eggleton P, Farndale RW (2002) Physical and functional interaction between cell-surface calreticulin and the collagen receptors integrin alpha2 beta1 and glycoprotein VI in human platelets. Thromb Haemost 88(4): 648-654. 